\title{
c-Kit mutation-positive advanced thymic carcinoma successfully treated as a mediastinal gastrointestinal stromal tumor: A case report
}

\author{
FUMIHIKO HIRAI, MAKOTO EDAGAWA, SHINICHIRO SHIMAMATSU, \\ RYO TOYOZAWA, GOUJI TOYOKAWA, KANAME NOSAKI, MASAFUMI YAMAGUCHI, \\ TAKASHI SETO, MITSUHIRO TWAKENOYAMA and YUKITO ICHINOSE
}

Department of Thoracic Oncology, National Kyushu Cancer Center, Fukuoka, Fukuoka 811-1395, Japan

Received October 2, 2015; Accepted January 20, 2016

DOI: $10.3892 / \mathrm{mco} .2016 .752$

\begin{abstract}
Thymic carcinoma is an exceptionally rare tumor, which has a very poor prognosis, differing from thymoma. Although cytotoxic chemotherapy is commonly used to treat advanced thymic carcinoma, its effectiveness has not been found to be sufficient. There are several reports that thymic carcinoma also harbors an oncogenic driver mutation, similar to lung cancer. A patient with a $c$-Kit mutation-positive thymic carcinoma received imatinib followed by sunitinib consecutively, which are both c-Kit inhibitors. Although the patient had achieved long-term disease control for 21 months, the primary lesion and pulmonary metastases had increased in size by November, 2014. Following failure of imatinib treatment, the patient received sunitinib, a multiple kinase inhibitor, initiated in December, 2014. Following administration of sunitinib, a computed tomography scan revealed a partial response and the disease was effectively controlled with continued sunitinib treatment for 6 months, up to June, 2015. The patient achieved long-term disease control ( 27 months) with imatinib followed by sunitinib. The efficacy of consecutive molecular-targeted therapy for thymic carcinoma was demonstrated in this case. Therefore, thymic carcinoma with oncogenic driver mutations should be treated with molecular-targeted agents rather than with cytotoxic drugs, and it may be suitable to treat $c$-Kit mutation-positive thymic carcinoma as a mediastinal gastrointestinal stromal tumor.
\end{abstract}

\section{Introduction}

Thymic carcinoma is an exceptionally rare tumor, which has a very poor prognosis, differing from thymoma. Advanced

Correspondence to: Dr Fumihiko Hirai, Department of Thoracic Oncology, National Kyushu Cancer Center, 3-1-1 Notame, Minami-ku, Fukuoka, Fukuoka 811-1395, Japan

E-mail: hirai.f@nk-cc.go.jp

Key words: thymic carcinoma, $c$-Kit mutation, imatinib, sunitinib, gastrointestinal stromal tumor thymic carcinoma is usually treated with chemotherapy using cytotoxic agents, such as anthracycline-based regimens, which are recommended by the National Comprehensive Cancer Network guidelines (www.nccn.org/professionals/physician_ gls/pdf/rectal.pdf). However, the efficacy of those regimens has not been satisfactory.

There have been several recent reports on the presence of an oncogenic driver mutation in thymic carcinoma, such as a $c$-Kit mutation (1-3). In adition, the efficacy of molecular-targeted therapy for thymic carcinoma has been demonstrated in previous case reports $(4,5)$. In the present case report, a patient with $c$-Kit mutation-positive thymic carcinoma was successfully treated with two consecutive c-Kit inhibitors.

\section{Case presentation}

A 35-year-old woman with a negative smoking history was diagnosed with advanced thymic carcinoma with multiple pulmonary metastases in April, 2011. The tumor was histologically diagnosed as squamous cell carcinoma. The patient received chemotherapy with carboplatin plus paclitaxel in May, 2011 and achieved a partial response. However, a computed tomography (CT) scan in March, 2012 revealed a relapse of the thymic carcinoma, with an increase in the size of the primary lesion and the pulmonary metastases. The patient then underwent treatment with c-MET and Akt inhibitors, as part of a clinical drug investigation. Despite treatment, the primary lesion and pulmonary metastases re-relapsed in March, 2013 after only a short period of stable disease. A repeat biopsy of the pulmonary metastases was performed to identify oncogenic driver mutations, such as those involving the epidermal growth factor receptor, echinoderm microtubule-associated protein-like 4-anaplastic lymphoma kinase and $c$-Kit genes, with the aim of identifying other potentially effective chemotherapies, particularly molecular-targeted therapies.

It was hypothesized that the pulmonary lesions included a metastatic lesion from the thymic $c$-Kit-expressing squamous cell carcinoma. This was confirmed by hematoxylin and eosin staining and immunohistochemistry (Fig. 1). Moreover, an active mutation of $c$-Kit in exon 11 (V559G) was detected in 


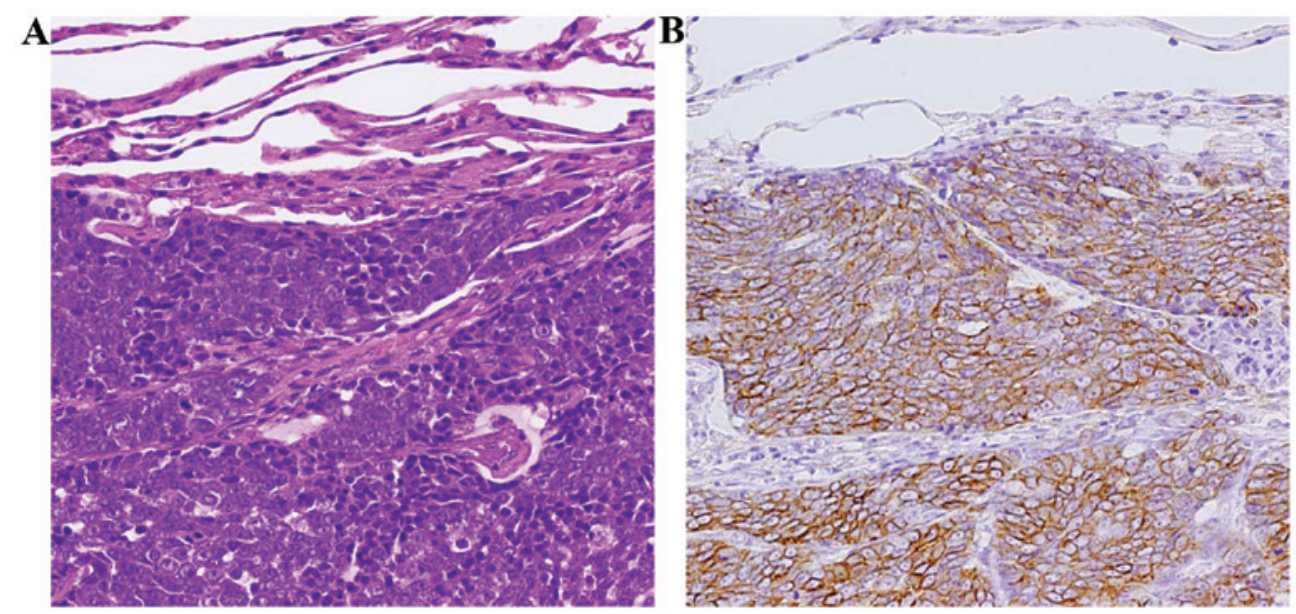

Figure 1. Histological examination of pulmonary metastases from the thymic carcinoma. (A) Hematoxylin and eosin staining (magnification, x200) and (B) immunohistochemical (IHC) examination for CD117 (c-Kit; magnification, x200). The other results of the IHC examination were as follows (not shown):

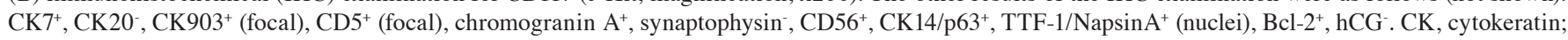
Bcl-2, B-cell lymphoma 2; hCG, human chorionic gonadotropin; TTF-1, thyroid transcription factor-1.
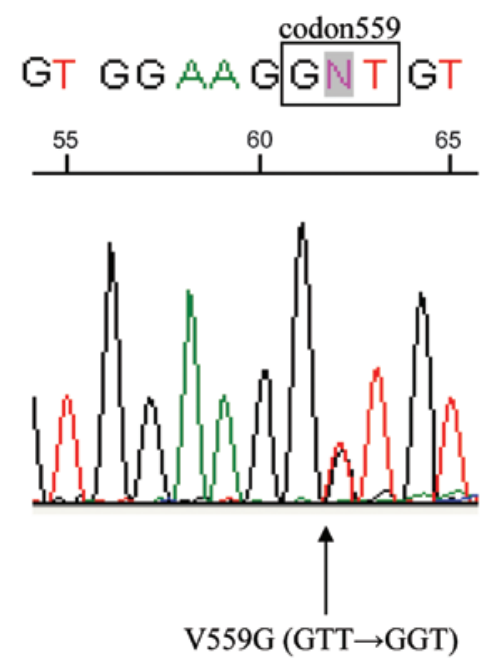

Figure 2. Gene sequence analysis of thymic carcinoma. A direct sequence analysis revealed a mutation of $c$-Kit (exon 11, V559G) in the pulmonary metastases from thymic carcinoma.
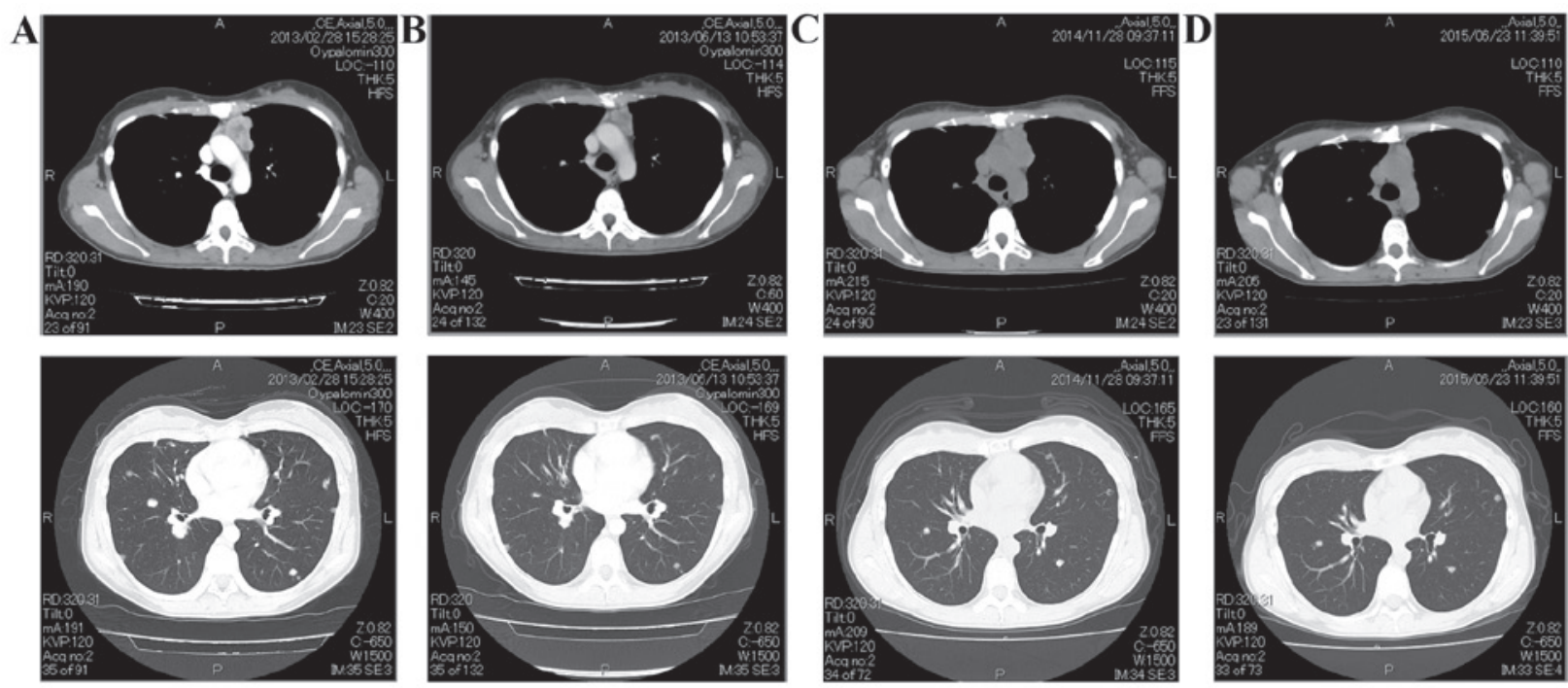

Figure 3. Chest computed tomography scans of the primary lesion and pulmonary metastases. (A) Prior to imatinib treatment; (B) following response to treatment with imatinib; (C) at the initiation of sunitinib treatment after a relapse during imatinib treatment; and (D) following response to imatinib. 
the pulmonary metastases using the reverse transcription polymerase chain reaction-invader assay (Fig. 2). No other driver mutations were detected. The patient was thus treated with imatinib, which is the standard treatment for gastrointestinal stromal tumors (GISTs).

Following treatment with imatinib, which was initiated in March, 2013, the primary lesion and pulmonary metastases exhibited a partial response (Fig. 3). Although long-term disease control was achieved for a period of 21 months, the size of the primary lesion and pulmonary metastases increased again in November, 2014. Following failure of imatinib treatment in December, 2014, the patient was treated with sunitinib, a multiple kinase inhibitor, which also inhibits c-Kit. A CT scan which was performed following administration of sunitinib revealed a partial response. In June, 2015, the patient's disease had been controlled with sunitinib for a period of 6 months, and the patient continued to receive sunitinib treatment, with a good performance status. Therefore, long-term disease control ( 27 months), was achieved with imatinib followed by sunitinib treatment.

\section{Discussion}

Several case reports recently demonstrated the presence of $c$-Kit mutations in thymic carcinoma $(1,2)$, with long-term disease control achieved with the c-Kit inhibitor imatinib (4-6). Simillary, there are several reports on the use of other molecular-targeted therapies for the treatment of thymic carcinoma, such as sorafenib and sunitinib (7-10). Molecular-targeted therapy for thymic carcinoma may only appear to achieve long-term stable disease. However, the correlation between oncogenic driver mutations and molecular-targeted agents remains unclear, with the exception of $c$-Kit mutations (11).

Patients with advanced GISTs determined to be $c$-Kit mutation-positive are generally treated with imatinib (12). Following failure and discontinuation of imatinib, GISTs have been treated with sunitinib as second-line therapy (13). The efficacy of these c-Kit inhibitors for $c$-Kit mutation-positive thymic carcinoma is reported to be similar $(5,14)$. Therefore, GISTs and $c$-Kit mutation-positive thymic carcinoma may share a similar background (15) and, thus, we should discuss the treatment strategies for thymic carcinoma as a GIST.

The efficacy of consecutive molecular-targeted therapies for thymic carcinoma was demonstrated in this case. Therefore, thymic carcinoma with an oncogenic driver mutation should be treated with molecular-targeted agents rather than with cytotoxic drugs, and it may be suitable to treat a $c$-Kit mutation-positive thymic carcinoma as a mediastinal GIST.

\section{References}

1. Yoh K, Nishiwaki Y, Ishii G, Goto K, Kubota K, Ohmatsu H, Niho S, Nagai K and Saijo N: Mutational status of EGFR and KIT in thymoma and thymic carcinoma. Lung Cancer 62: 316-320, 2008

2. Petrini I, Zucali PA, Lee HS, Pineda MA, Meltzer PS, Walter-Rodriguez B, Roncalli M, Santoro A, Wang Y and Giaccone G: Expression and mutational status of c-kit in thymic epithelial tumors. J Thorac Oncol 5: 1447-1453, 2010.

3. Aisner SC, Dahlberg S, Hameed MR, Ettinger DS, Schiller JH, Johnson DH, Aisner J and Loehrer PJ: Epidermal growth factor receptor, C-kit and Her2/neu immunostaining in advanced or recurrent thymic epithelial neoplasms staged according to the 2004 World Health Organization in patients treated with octreotide and prednisone: An Eastern Cooperative Oncology Group study. J Thorac Oncol 5: 885-892, 2010.

4. Ströbel P, Hartmann M, Jakob A, Mikesch K, Brink I, Dirnhofer S and Marx A: Thymic carcinoma with overexpression of mutated KIT and the response to imatinib. N Engl J Med 350: 2625-2626, 2004.

5. Buti S, Donini M, Sergio P, Garagnani L, Schirosi L, Passalacqua R and Rossi G: Impressive response with imatinib in a heavily pretreated patient with metastatic c-KIT mutated thymic carcinoma. J Clin Oncol 29: e803-e805, 2011.

6. Hamada S, Masago K, Mio T, Hirota S and Mishima M: Good clinical response to imatinib mesylate in atypical thymic carcinoid with KIT overexpression. J Clin Oncol 29: e9-e10, 2011.

7. Disel U, Oztuzcu S, Beșen AA, Karadeniz C, Köse F, Sümbül AT, Sezer A, Nursal GN, Abalı H and Ozyılkan O: Promising efficacy of sorafenib in a relapsed thymic carcinoma with C-KIT exon 11 deletion mutation. Lung Cancer 71: 109-112, 2011.

8. Li XF, Chen Q, Huang WX and Ye YB: Response to sorafenib in cisplatin-resistant thymic carcinoma: A case report. Med Oncol 26: 157-160, 2009.

9. Thomas A, Rajan A, Berman A, Tomita Y, Brzezniak C, Lee MJ, Lee S, Ling A, Spittler AJ, Carter CA, et al: Sunitinib in patients with chemotherapy-refractory thymoma and thymic carcinoma: An open-label phase 2 trial. Lancet Oncol 16: 177-186, 2015.

10. Neuhaus T and Luyken J: Long lasting efficacy of sorafenib in a heavily pretreated patient with thymic carcinoma. Target Oncol 7: 247-251, 2012.

11. Pagano M, Sierra NM, Panebianco M, Rossi G, Gnoni R, Bisagni $\mathrm{G}$ and Boni C: Sorafenib efficacy in thymic carcinomas seems not to require c-KIT or PDGFR-alpha mutations. Anticancer Res 34: 5105-5110, 2014.

12. Demetri GD, von Mehren M, Blanke CD, Van den Abbeele AD, Eisenberg B, Roberts PJ, Heinrich MC, Tuveson DA, Singer S, Janicek M, et al: Efficacy and safety of imatinib mesylate in advanced gastrointestinal stromal tumors. N Engl J Med 347: 472-480, 2002.

13. Demetri GD, van Oosterom AT, Garrett CR, Blackstein ME, Shah MH, Verweij J, McArthur G, Judson IR, Heinrich MC, Morgan JA, et al: Efficacy and safety of sunitinib in patients with advanced gastrointestinal stromal tumour after failure of imatinib: A randomised controlled trial. Lancet 368: 1329-1338, 2006.

14. Lim SH, Lee JY, Sun JM, Kim KM, Ahn JS, Ahn MJ and Park K: A new KIT gene mutation in thymic cancer and a promising response to imatinib. J Thorac Oncol 8: e91-e92, 2013.

15. Rossi V, Donini M, Sergio P, Passalacqua R, Rossi G and Buti S: When a thymic carcinoma 'becomes' a GIST. Lung Cancer 80: 106-108, 2013. 